\title{
EDITORIAL
}

\section{The Combination of Theory and Practice as well as Finance and Insurance is a successful formula}

\begin{abstract}
Astin meetings are held in high regard and affection by the attendees; both academics and practitioners. There is a history of regular attendance, which indicates the value the participants place on the meetings. The Astin Bulletin has an excellent reputation in the insurance industry and its articles are regularly cited and continuously referred to by other journals. However there is a school of thought that it is all too theoretical and is therefore of limited interest to the practising actuary. As a practising actuary, I have not found this to be the case and without fail find practical ideas and applications from each Astin meeting I attend and also each edition of the Astin Bulletin I read.
\end{abstract}

\section{THE IMPORTANCE OF THEORY}

Actuaries need to have a sound theoretical base to undertake their work. This is particularly true of the general insurance and investment fields. It is very easy, in both these areas, to believe that one is undertaking sound analyses while actually making serious mistakes or performing sub-optimally if one is not up to date with all the theoretical developments. It is important in this context to realise that 'You don't know what you don't know'. Unfortunately this is all too common among actuaries who pride themselves on their practicality.

DFA is a case in point. It is regarded as a very practical subject, but it is surprising the number of DFA practitioners who have not kept up with recent developments. The use of copulas to handle tail dependency rather than naively assuming independence, has a major impact on capital allocation. This theory is not new, though the ability to use it computationally is a phenomenon of the reduction in the cost of computing power. Consequently, it has recently become much more useful to the practitioner. There have been a number of Astin papers that have analysed the application of copulas further and also adapted them to modern computing technology including covering many of the practical issues of choosing which copula to use.

Some of the developments in the theory of risk measures are also very relevant to capital allocation and DFA. The theory can seem very abstract but the use of non-coherent risk measures can dramatically distort the results and cause wrong decisions to be made. A good example is the use of Var as a risk measure. It is widely used in the banking world for market risk and where work on capital allocation preceded that in the insurance industry. Consequently there is a temptation in diversified financial groups to use Var as a capital allocation tool for insurance risks. When that is done, it is usually with disastrous results as there will often be an understatement of the capital requirements for the insurance risks. Without understanding the theoretical reasons as to why 
this occurs is a recipe for disaster. Var works for banks for market risk because the risks are largely symmetrical. Under these circumstances Var will order risks in the same way as a number of coherent risk measures. However this is not the case with skew risks, especially if there are tail dependencies. This is usually the situation for insurance companies and also for operational risk. Some very large financial institutions have made some serious mistakes as a result of not being aware of the relevant theory. For the individual actuary there is the real risk of a career ending in tears by not being up to speed with the theory. There are also very serious implications for the profession as a whole.

Another development is pricing methodology. The work by Wang, Panjer et al is of major importance in providing consistent ways of pricing across markets and perhaps even more practically for measuring the relative attractiveness of different markets or of measuring the underwriting cycle. A major practical problem is how to maintain consistency within an organisation. The Wang transform provides an approach that allows practical and readily implementable methodology to solve an insurance company management problem that would otherwise be an extremely complex organisational issue.

Often the practical actuary can utilise what superficially seem to be very theoretical papers. For example, practising actuaries are not usually interested in probabilities of ruin and hence may be tempted to dismiss the many papers dealing with approximations to it to be merely of interest to the academics. However these formulae also provide an approximation to the claim frequency on an excess of loss contract. Simple appreciation of this fact allows the practising actuary to utilise the work in this area and thus streamline the pricing. I have found judicial reading of such articles to be a fruitful source of ideas.

\section{ASTIN WELCOMES PRACTICAL PAPERS}

The Astin Bulletin would welcome more papers by practising actuaries. Indeed this would create a virtuous circle of creating more interest in the application of theoretical developments which in turn would encourage more academic work in this area and also more practising actuaries to participate in Astin.

It would also encourage academicians to solve problems that were complex but for which practically important. Assumptions that are mathematically convenient or elegant are not helpful to the practitioner. Solutions to problems that are very complex are invaluable. The academics can identify fruitful areas for them to research by understanding where the problems lie for the practitioner. In any event, actuarial science is an applied science and therefore by definition must have its roots in the real world and produce relevant applications.

However it is important that Astin does not let its academic standards slip, especially in the Bulletin. It is important that it has a high reputation for academic rigour not only to maintain its reputation and attract academics to publish articles. The two are not incompatible and also demonstrate to the practitioner the importance of correct theory. However the discussions at meetings cover many more of the practical aspects. This is not surprising and a forum for transmitting these to a wider audience would be of value to the whole profession. 


\section{CONVERGENCE OF FINANCIAL MARKETS}

The Astin Bulletin now incorporates the Journal of the Afir Section of the IAA. This is not just an administrative convenience or a consequence of banking and insurance converging. It is also of immense use to the practising and academic actuary alike as it allows ideas in one area to be more easily utilised in the other. Furthermore an analysis of the different techniques provides some insights into the differences of the two areas. For example, much of the pricing methodology in general insurance is based on the assumption that the risk will be held on the balance sheet of the insurer and not traded( there is no real market place or natural short sellers for most insurance risks). However the banking approach assumes a market based approach and the corresponding pricing methodologies. This requires markets to exist and the ability to diversify risk. This is not possible if the risks are too large for the number of participants in the market place. Hence the 'insurance pricing models' will be required with corresponding charges for risk. However for something that can be readily diversified, then it is likely that a market based risk charge can apply. This also has implications for the relative pricing of reinsurance versus insurance.

Diversification by risk and also by risk class is something that the actuary can learn from the different approaches. Financial risks usually have significant correlations and/or tail dependencies that diversification is used to mitigate. However this is often not the case between physical risks. Thus earthquake risk is only correlated across zones and not world wide whereas there are world wide correlations on credit risks and hence the problems that some insurers ran into in recent years when they did not realise this.

Another area is risk. Many insurance company actuaries (and indeed many nor-actuaries) intuitively believe that high risk should be handled by high discount rates. They do not understand that this gives the wrong answers and the need for risk neutral probabilities, martingales etc. This is an issue not just of theory but of being exposed to different techniques. It is also an area where incorrect methodology gives rise to wrong decisions and is heavily biased towards long term projects.

\section{The Future}

I believe that the combination of academic and practitioner, of finance and insurance is a powerful one. Provided we can all co-operate and communicate together the future is bright and that we will all benefit from each other and that the sum of the parts is much greater than the whole. Failure to keep up with modern developments even if they seem abstruse, mathematically complicated and difficult to apply in practice is not a sign of a lack of practicality but more of a high risk strategy to both the individual and the profession. 